\title{
A lattice Boltzmann method for a two-phase flow containing solid bodies with viscoelastic membranes
}

\author{
Masato Yoshino ${ }^{1,2, a}$ and Toshiro Murayama ${ }^{1}$ \\ 1 Department of Mechanical Systems Engineering, Shinshu University \\ 4-17-1, Wakasato, Nagano-shi, Nagano 380-8553, Japan \\ 2 CREST, Japan Science and Technology Agency \\ 4-1-8, Honcho, Kawaguchi-shi, Saitama 332-0012, Japan
}

\begin{abstract}
A lattice Boltzmann method (LBM) for two-phase flows containing solid bodies with viscoelastic membranes is proposed. The method is based on the two-phase LBM, in which one phase is regarded as the solid phase. In the present model, the membrane is assumed to be composed of identical particles that are connected to their neighboring particles by elastic springs to take account of stretching and compression effects. The method is applied to two representative problems, namely the behavior of a viscoelastic body under shear flow and the motion of a viscoelastic body in a Poiseuille flow. Tank-tread motion and axial migration, which are both characteristic of the motion of viscoelastic bodies, are simulated by using the method. These results indicate that the method is capable of simulating the complex behavior of viscoelastic bodies in capillaries, such as the motion of red blood cells in blood flows.
\end{abstract}

\section{Introduction}

Problems of solid-fluid two-phase flow can be found not only in engineering applications, such as solid-liquid slurry flow, but also in biological fields connected with blood flow in capillaries. If the latter problem is taken as an example, the interaction between red blood cells (RBCs) and blood plasma becomes important in small blood vessels where the cellular size is comparable to the vessel diameter. A normal RBC has a biconcave shape that is easily deformed into ellipsoidal shape under uniform shear stress. The deformability of the RBC is related to the erythrocyte configuration, the viscosity of the internal fluid, and the viscoelasticity of the membrane $[1,2]$. In particular, the elastic behavior of the RBC is determined by the nature of the elastic membrane. Although investigations of the complicated behavior of the RBC are needed, it is difficult to examine the phenomena that are involved, particularly in microscale vessels, by means of experiments. Therefore, numerical simulation is considered to be an effective approach for microscopic investigation of such flow problems.

With regard to numerical studies of solid-fluid two-phase flows, Boryczko et al. [3] and Dzwinel et al. [4] have proposed discrete particle models for simulation of RBCs in capillary vessels by the Lagrangian coordinates technique. Tanaka and Takano [5] used smoothed particle hydrodynamics $[6,7]$ to investigate the microscopic behavior of blood flow. Tsubota et al. [8] also carried out a simulation based on the particle method [9] to examine a peculiar rotary motion (i.e., tank-tread motion [10,11]). Although these studies have produced interesting results, because of the complexity of the algorithms employed for the solid-fluid coupling problems, elaborate computing code is required and computation times are rather long.

\footnotetext{
${ }^{a}$ e-mail: masato@shinshu-u.ac.jp
} 


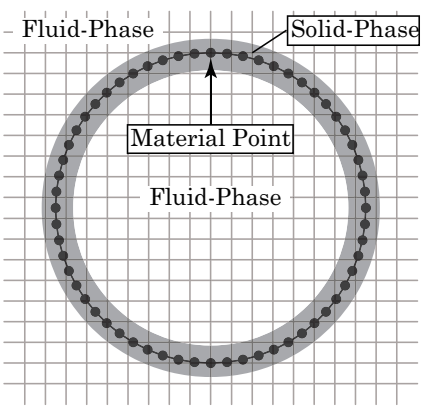

Fig. 1. Membrane model.

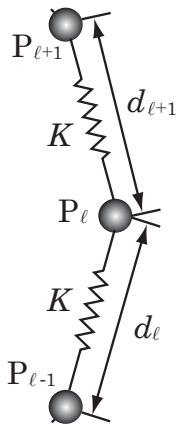

Fig. 2. Spring system with material points $\mathrm{P}_{\ell-1}, \mathrm{P}_{\ell}$, and $\mathrm{P}_{\ell+1} . K$ is an elastic modulus and $d_{\ell}$ is a length of the elastic spring between $\mathrm{P}_{\ell}$ and $\mathrm{P}_{\ell-1}$.

Recently, the lattice Boltzmann method (LBM) [12-14] has been developed into an alternative and promising numerical scheme for simulating flows of viscous fluids and multicomponent or multiphase fluids. In particular, for solid-fluid two-phase flows, Ladd [15-17] was the first to simulate solid-fluid suspensions of spheres in shear flows. Aidun and Lu [18] and Qi [19,20] also performed similar simulations for cylinders and for nonspherical particles, respectively. Inamuro et al. [21] investigated the motion of neutrally buoyant circular cylinders between parallel walls by using the LBM. These are confined to simulations involving rigid bodies; the LBM has not previously been thoroughly established for two-phase flows containing viscoelastic bodies.

We proposed an LBM for flows containing solid bodies in the form of viscoelastic membranes by regarding the membrane of the $\mathrm{RBC}$ as an elastic body with a finite thickness. By using the method, we simulated the behavior of a viscoelastic body under shear flows and the motion of a viscoelastic body in a plane Poiseuille flow.

\section{Numerical Method}

As described previously [22], we use nondimensional variables defined by a characteristic length $L$, a characteristic particle speed $c$, a characteristic time scale $t_{0}=L / U$, where $U$ is a characteristic flow speed, a reference order parameter $\phi_{0}$, and a reference density $\rho_{0}$. The two-dimensional nine-velocity model is used in the present study. The velocity vectors in the nine-velocity model are given by $\boldsymbol{c}_{1}=\mathbf{0}, \boldsymbol{c}_{i}=[\cos (\pi(i-2) / 2, \sin (\pi(i-2) / 2](i=2,3,4,5)$, and $\boldsymbol{c}_{i}=\sqrt{2}[\cos (\pi(i-11 / 2) / 2, \sin (\pi(i-11 / 2) / 2](i=6,7,8,9)$.

\subsection{Formulation}

The numerical algorithm is based mainly on the LBM for incompressible two-phase flows with large density differences, as proposed by Inamuro et al. [22] Hence, for a detailed formulation of the present method, please refer to the literature and to the recently published Ref. [23]. We assume that the fluid phase is an inelastic Newtonian fluid and that the solid phase is a membrane composed of identical particles that are connected to their neighbors by elastic springs. Also, the interior phase surrounded by the membrane is assumed to be the same Newtonian fluid as the fluid outside the membrane.

\subsection{Introduction of Elastic Force}

Because the viscous effect of the body is inherent in the original LBM for two-phase flows, only an elastic force based on the Kelvin-Voigt model needs to be introduced into the LBM. As 


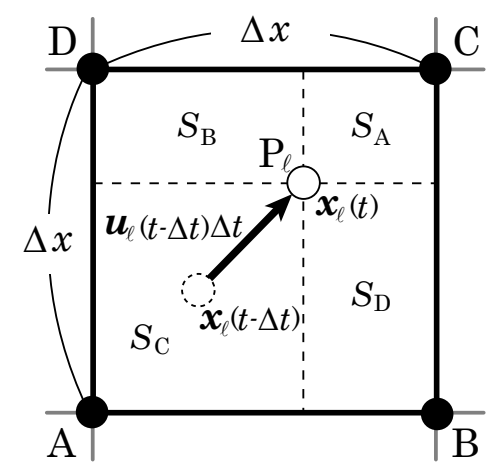

Fig. 3. Dynamic behavior of material point $\mathrm{P}_{\ell}$ and the surrounding four grids, $\mathrm{A}, \mathrm{B}, \mathrm{C}$, and $\mathrm{D}$.

shown in Fig. 1, a two-dimensional annular membrane with a finite thickness is considered. It is assumed that the membrane consists of $M$ particles positioned a distance $d_{0}$ apart and that these particles are initially arranged at the midpoint in the thickness direction of the membrane. Also, the particles are connected by identical elastic springs that have an initial length $d_{0}$ and an elastic modulus $K$; hereafter, we refer to such particles as "material points."

Here, let us consider the arrangement between the material points $\mathrm{P}_{\ell+1}, \mathrm{P}_{\ell}$, and $\mathrm{P}_{\ell-1}(\ell=$ $1,2, \ldots, M)$ as shown in Fig. 2. Note that $\mathrm{P}_{0}=\mathrm{P}_{M}$ and $\mathrm{P}_{M+1}=\mathrm{P}_{1}$, because of the circular arrangement of the material points. If we focus on the material point $\mathrm{P}_{\ell}$ and apply Hooke's law to the spring system, the elastic force $\boldsymbol{F}_{\ell}$ is given by the following equation:

$$
\begin{aligned}
\boldsymbol{F}_{\ell} & =\boldsymbol{F}_{\ell+}-\boldsymbol{F}_{\ell-} \\
& =K d_{\ell+1} \frac{\boldsymbol{x}_{\ell+1}-\boldsymbol{x}_{\ell}}{\left|\boldsymbol{x}_{\ell+1}-\boldsymbol{x}_{\ell}\right|}-K d_{\ell} \frac{\boldsymbol{x}_{\ell}-\boldsymbol{x}_{\ell-1}}{\left|\boldsymbol{x}_{\ell}-\boldsymbol{x}_{\ell-1}\right|},
\end{aligned}
$$

where $d_{\ell}$ is the stretch/compression displacement between the two material points $\mathrm{P}_{\ell}$ and $\mathrm{P}_{\ell-1}$, $\boldsymbol{x}_{\ell}$ is the position vector, and $\left|\boldsymbol{x}_{\ell}\right|$ is the magnitude of $\boldsymbol{x}_{\ell}$. The dynamic behavior of the material points is as follows. As shown in Fig. 3, during the time step $\Delta t$, the material point $\mathrm{P}_{\ell}$ at the position $\boldsymbol{x}_{\ell}(t-\Delta t)$ and at time $t-\Delta t$ moves to the position given by the following equation:

$$
\boldsymbol{x}_{\ell}(t)=\boldsymbol{x}_{\ell}(t-\Delta t)+\boldsymbol{u}_{\ell}(t-\Delta t) \Delta t
$$

where $\boldsymbol{u}_{\ell}(t-\Delta t)$ is the velocity of the material point, which is obtained by linear interpolation of the velocities at the surrounding four grids, A, B, C, and D. At the same time, the displacement of the springs is changed, and the material point is subject to an elastic force $\boldsymbol{F}_{\ell}$, as given by Eq. (1). In addition, because the position of the material point does not coincide with computational grids in general, the redistribution of the elastic force at $\mathrm{P}_{\ell}$ to the surrounding grids is carried out by a linear extrapolation method. In Fig. 3, for example, the elastic forces $\boldsymbol{F}_{\ell}(\Psi)$ at the surrounding four grids $\Psi(=\mathrm{A}, \mathrm{B}, \mathrm{C}, \mathrm{D})$ are given by

$$
\boldsymbol{F}_{\ell}(\Psi)=\frac{S_{\Psi}}{(\Delta x)^{2}} \boldsymbol{F}_{\ell} \quad \text { for } \Psi=\mathrm{A}, \mathrm{B}, \mathrm{C}, \mathrm{D},
$$

where $\Delta x$ is the spacing of the square lattice, and $S_{\mathrm{A}}, S_{\mathrm{B}}, S_{\mathrm{C}}$, and $S_{\mathrm{D}}$ are the respective areas of the rectangle shown in Fig. 3. Thus, the net elastic force at the grid $\Psi$ can be obtained by the sum of contributions from the related material points.

The above-mentioned elastic force is incorporated into the equation of motion. In the computations, the term $3 E_{i} F_{\alpha} c_{i \alpha} / \rho_{\mathrm{S}}$ is added to the function $g_{i}^{\mathrm{c}}$ for calculation of the predicted velocity in the solid phase alone (see Eq. (34) in Ref. [23]), where $E_{i}$ is the constant coefficient, $\rho_{\mathrm{S}}$ is the density of the solid phase, and $\alpha, \beta=x, y$ (subscripts $\alpha$ and $\beta$ represent Cartesian coordinates and the summation convention is used). 


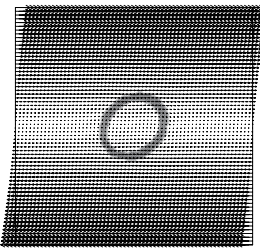

(a) $t^{*}=0.610$

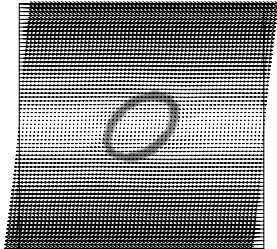

(b) $t^{*}=0.916$

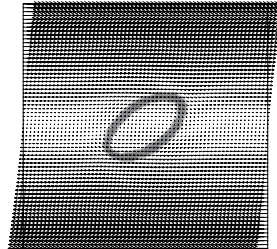

(c) $t^{*}=1.22$

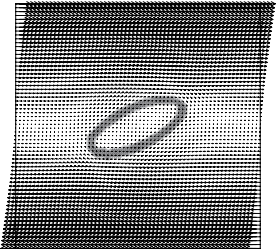

(d) $t^{*}=3.05$

Fig. 4. Time evolutions of body shape and velocity vectors for $K \Delta x=1 \times 10^{-5}$ at $\operatorname{Re}=0.156 . t^{*}=t \Gamma$ is the dimensionless time.

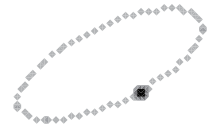

(a) $t^{*}=2.44$

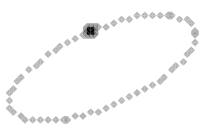

(c) $t^{*}=14.6$

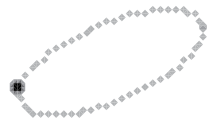

(b) $t^{*}=9.77$

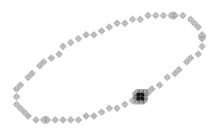

(d) $t^{*}=25.0$

Fig. 5. Unsteady rotary motion of membrane for $K \Delta x=1 \times 10^{-5}$ at $\operatorname{Re}=0.156$. The black circle indicates a certain material point in the membrane $\left(t^{*}=t \Gamma\right)$.

\section{Numerical Examples}

\subsection{Motion of a Body under Shear Flow}

To calculate the behavior of an annular body composed of a viscoelastic membrane under shear flow, we consider a square domain with sides $L_{x}=L_{y}=128 \Delta x$. A viscoelastic annular body is placed at the center of this domain. The membrane is assumed to be composed of 60 identical material points $(M=60)$, and the thickness of the membrane is approximately $6 \Delta x$. The initial distance between the centroid and each material point is set to be $r=16 \Delta x$. The body is brought to the equilibrium state at rest and at $t=0$, the top and bottom walls begin to move with velocities $u_{\mathrm{w}}$ and $-u_{\mathrm{w}}$, respectively. The no-slip boundary condition is used on the moving walls, and the periodic boundary condition is used on the lateral sides of the domain. Note that, in order to examine the elastic effect itself, surface tension is assumed to be absent. The Reynolds number $\operatorname{Re}=\Gamma r^{2} / \nu_{\mathrm{F}}$ is 0.156 , where $\Gamma=2 u_{\mathrm{w}} / L_{y}$ is the shear rate and $\nu_{\mathrm{F}}$ is the kinematic viscosity of the fluid.

First, the time evolutions of the body shape and velocity vectors for $K \Delta x=1 \times 10^{-5}$ are shown in Fig. 4, where the gray region represents the viscoelastic membrane. Note that the dimensionless time is defined by $t^{*}=t \Gamma$. It is found that the body is stretched out and becomes deformed into an elongated ellipsoidal shape as time passes.

Next, the motion of the membrane is investigated. Figures 5 and 6 show the unsteady behavior of the membrane and the temporal position of a certain material point, respectively. From these figures, it is found that the membrane performs an ellipsoidal rotation with a constant period $\left(\approx 23 t^{*}\right)$. This periodic motion is generally called the tank-tread motion [10, 11], which is a rotary motion like that of a caterpillar track. Thus, an interesting phenomenon characteristic of RBC behavior can be simulated by using the present method. 
Wall

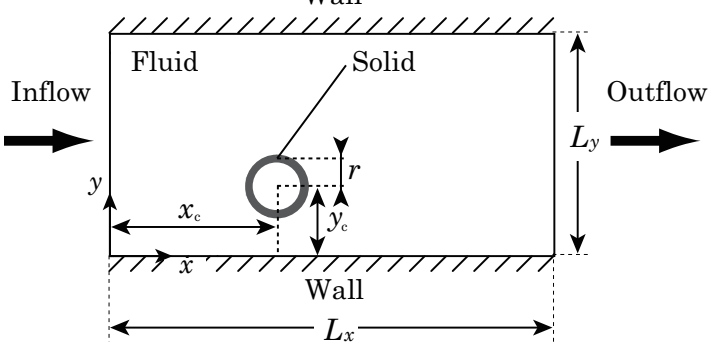

Fig. 7. Computational domain of Poiseuille flow problem and initial position of viscoelastic body.

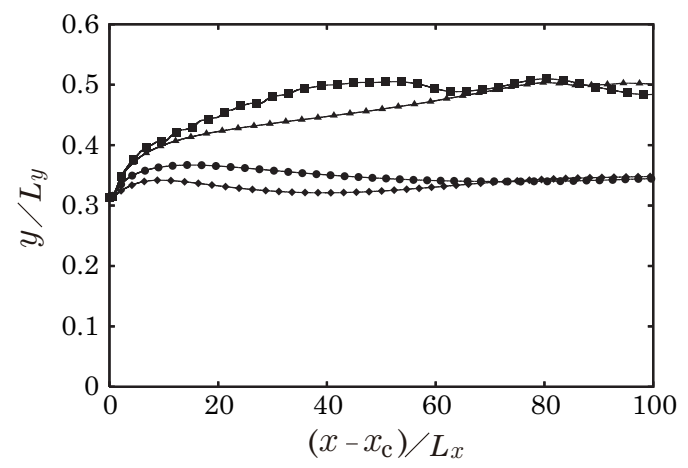

Fig. 9. Lateral migration curves of bodies with various elastic moduli $(\mathbf{\square}, K \Delta x=5 \times$ $10^{-5} ; \boldsymbol{\Delta}, K \Delta x=1 \times 10^{-4} ; \quad \bullet, K \Delta x=$ $\left.5 \times 10^{-4} ; \diamond, K \Delta x=1 \times 10^{-3}\right)$ at $\operatorname{Re}=25.6$. These bodies are released from the same initial vertical position $\left(y_{c} / L_{y}=0.313\right)$.

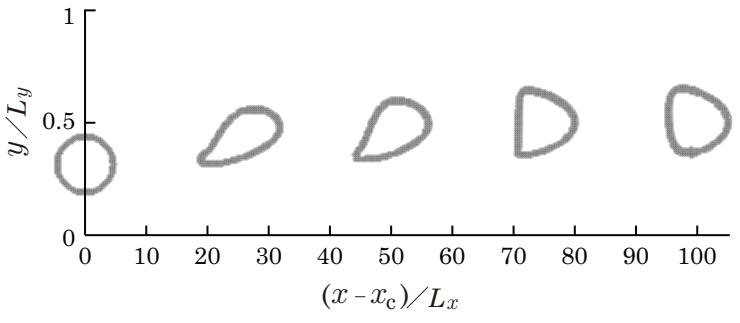

Fig. 8. Time variations of shape and position of body for $K \Delta x=1 \times 10^{-4}$ at $\operatorname{Re}=25.6$.

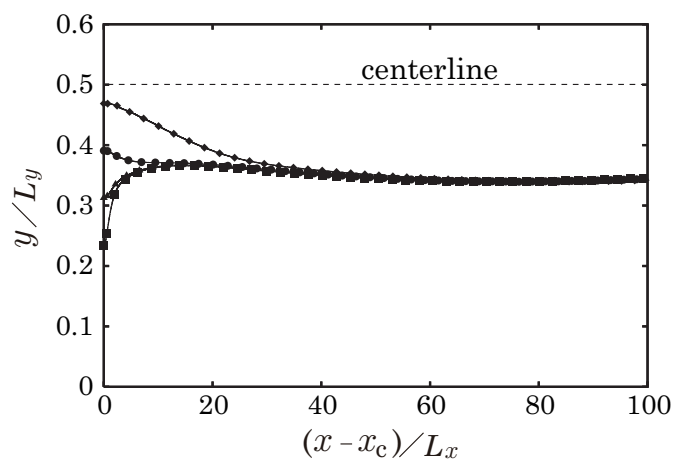

Fig. 10. Lateral migration curves of bodies with an elastic modulus ( $K \Delta x=5 \times 10^{-4}$ ) at $\operatorname{Re}=25.6$. The initially released vertical positions are as follows: $\mathbf{\square}, 0.234$; $\mathbf{\Delta}, 0.316$; $\bullet, 0.391 ; \diamond, 0.469$. The equilibrium position is $y_{\mathrm{c}} / L_{y} \approx 0.35$ in all cases.

\subsection{Motion of a Body in a Poiseuille Flow}

To calculate the behavior of a viscoelastic body in a Poiseuille flow, we consider a rectangular domain with $L_{x} \times L_{y}=256 \Delta x \times 128 \Delta x$, as shown in Fig. 7 . The same viscoelastic annular body as discussed in the previous problem is placed at $\left(x_{\mathrm{c}} / L_{x}, y_{\mathrm{c}} / L_{y}\right)=(0.375,0.313)$, where $\left(x_{\mathrm{c}}, y_{\mathrm{c}}\right)$ is the position of the centroid of the body. In this problem, the elastic modulus of the body is changed in the range of $10^{-5} \leq K \Delta x \leq 10^{-3}$. The no-slip boundary condition is used on the top and bottom walls, and the periodic boundary condition with a pressure difference is used at the inlet and outlet. The pressure difference is set to $\Delta p=1 \times 10^{-5}$. The density ratio of the solid to the fluid is set to 1.04 , which corresponds to the ratio of the density of a $\mathrm{RBC}$ to that of blood plasma in a typical human being. Also, the kinematic viscosities of the solid and the fluid are assumed to be equal to one another. The gravitational effect is neglected; it follows that a neutrally buoyant flow is assumed in this simulation. The Reynolds number $\operatorname{Re}=2 r u_{\max } / \nu_{\mathrm{F}}$ is fixed at 25.6, with $u_{\max }$ being the maximum velocity at the centerline between the walls.

First, the effect of elasticity on the motion of the body is investigated. As an example, the shape and position of the body for $K \Delta x=1 \times 10^{-4}$ are shown in Fig. 8. It can be seen that the body migrates laterally with an almost flat shape on the upstream side and a convex shape on the downstream side after transitional flows. Figure 9 shows the lateral migration curves of bodies with various elastic moduli $\left(K \Delta x=5 \times 10^{-5}, 1 \times 10^{-4}, 5 \times 10^{-4}\right.$, and $\left.1 \times 10^{-3}\right)$ from the same initial vertical position $\left(y_{\mathrm{c}} / L_{y}=0.313\right)$. It can be seen that the body migrates toward the centerline in the early state, and then reaches an equilibrium lateral position. This 
equilibrium position can be classified into two types; for a low elasticity, it is very close to the centerline (which is called axial migration), whereas for a larger elasticity, it is at a certain position between the centerline and the bottom wall.

Next, in the case of $K \Delta x=5 \times 10^{-4}$, the lateral migration curves for a body initially released at various positions between the centerline and the bottom wall are shown in Fig. 10. Regardless of the initial position, the body migrates to almost the same equilibrium position $\left(y_{\mathrm{c}} / L_{y} \approx 0.35\right)$, which is closer to the centerline than the equilibrium position $\left(y_{\mathrm{c}} / L_{y}=0.265\right)$ for the rigid body at $\mathrm{Re}=26.7$, as reported by Inamuro et al. [21] Segré and Silberberg [24] showed experimentally that neutrally buoyant particles in a pipe finally reach a certain equilibrium lateral position. Thus, a peculiar phenomenon similar to the Segré-Silberberg effect is found in the present simulations.

\section{Concluding Remarks}

A lattice Boltzmann method for two-phase flows containing solid bodies with viscoelastic membranes is proposed. The method is applied to two representative problems, namely the behavior of a viscoelastic body under shear flow and the motion of a viscoelastic body in a Poiseuille flow. Although the extension of the method to three-dimensions and quantitative investigations are required in future work, the method could be a promising approach for simulating the complex behavior of viscoelastic bodies in capillaries, such as the motion of red blood cells in blood flows.

This work is supported by the Grant-in-Aid for Young Scientists (B) [No.18760121] of the Ministry of Education, Culture, Sports, Science, and Technology, Japan (MEXT) and of the Japan Society for the Promotion of Science (JSPS).

\section{References}

1. T. Shiga, N. Maeda, K. Kon, Crit. Rev. Oncol. Hematol. 10, 9 (1990)

2. N. Maeda, T. Shiga, J. Blood Rheol. 7, 3 (1993)

3. K. Boryczko, W. Dzwinel, D.A. Yuen, J. Mol. Model. 9, 16 (2003)

4. W. Dzwinel, K. Boryczko, D.A. Yuen, J. Colloid Interface Sci. 258, 163 (2003)

5. N. Tanaka, T. Takano, Int. J. Comput. Methods 2, 555 (2005)

6. J.J. Monaghan, Comput. Phys. Commun. 48, 89 (1988)

7. J.J. Monaghan, J. Comput. Phys. 110, 399 (1994)

8. K. Tsubota, S. Wada, T. Yamaguchi, Comput. Methods Programs Biomed. 83, 139 (2006)

9. S. Koshizuka, Comput. Fluid Dyn. J. 4, 29 (1995)

10. T.M. Fischer, H.S. Schönbein, Blood Cells 3, 351 (1977)

11. T.M. Fischer, Biophys. J. 32, 863 (1980)

12. R. Benzi, S. Succi, M. Vergassola, Phys. Rep. 222, 145 (1992)

13. S. Chen, G.D. Doolen, Annu. Rev. Fluid Mech. 30, 329 (1998)

14. S. Succi, The Lattice Boltzmann Equation for Fluid Dynamics and Beyond (Oxford University Press, Oxford, 2001)

15. A.J.C. Ladd, J. Fluid Mech. 271, 285 (1994)

16. A.J.C. Ladd, Phys. Rev. Lett. 76, 1392 (1996)

17. A.J.C. Ladd, Phys. Fluids 9, 491 (1997)

18. C.K. Aidun, Y. Lu, J. Stat. Phys. 81, 49 (1995)

19. D. Qi, Int. J. Mod. Phys. C 8, 985 (1997)

20. D. Qi, J. Fluid Mech. 385, 41 (1999)

21. T. Inamuro, K. Maeba, F. Ogino, Int. J. Multiphase Flow 26, 1981 (2000)

22. T. Inamuro, T. Ogata, S. Tajima, N. Konishi, J. Comput. Phys. 198, 628 (2004)

23. T. Inamuro, Fluid Dyn. Res. 38, 641 (2006)

24. G. Segré, A. Silberberg, Nature 189, 209 (1961) 